\title{
Solutions for a Singular Hadamard-Type Fractional Differential Equation by the Spectral Construct Analysis
}

\author{
Xinguang Zhang $\mathbb{D}^{1,2}$ Lixin Yu $\mathbb{D},{ }^{1}$ Jiqiang Jiang $\mathbb{D}^{3},{ }^{3}$ Yonghong $W u\left(\mathbb{D},{ }^{2}\right.$ and Yujun Cui $\mathbb{C}^{4}$ \\ ${ }^{1}$ School of Mathematical and Informational Sciences, Yantai University, Yantai, 264005 Shandong, China \\ ${ }^{2}$ Department of Mathematics and Statistics, Curtin University of Technology, Perth, WA 6845, Australia \\ ${ }^{3}$ School of Mathematical Sciences, Qufu Normal University, Qufu, 273165 Shandong, China \\ ${ }^{4}$ Department of Mathematics, Shandong University of Science and Technology, Qingdao, 266590 Shandong, China
}

Correspondence should be addressed to Xinguang Zhang; zxg123242@163.com and Lixin Yu; lixinyu970@gmail.com

Received 2 June 2020; Accepted 5 August 2020; Published 30 September 2020

Academic Editor: Gisele Mophou

Copyright (c) 2020 Xinguang Zhang et al. This is an open access article distributed under the Creative Commons Attribution License, which permits unrestricted use, distribution, and reproduction in any medium, provided the original work is properly cited.

\begin{abstract}
In this paper, we consider the existence of positive solutions for a Hadamard-type fractional differential equation with singular nonlinearity. By using the spectral construct analysis for the corresponding linear operator and calculating the fixed point index of the nonlinear operator, the criteria of the existence of positive solutions for equation considered are established. The interesting point is that the nonlinear term possesses singularity at the time and space variables.
\end{abstract}

\section{Introduction}

In this paper, we focus on the existence of positive solutions for the following singular Hadamard-type fractional differential equation:

$$
\left\{\begin{array}{l}
\mathscr{D}_{\mathbf{t}}^{\alpha} \mathscr{D}_{\mathbf{t}}^{\beta} z(t)=f\left(t, z(t),-\mathscr{D}_{\mathbf{t}}^{\beta} z(t)\right), 1<t<e, \\
z(1)=\sigma z(1)=\sigma z(e)=0, \mathscr{D}_{\mathbf{t}}^{\beta} z(1)=\sigma \mathscr{D}_{\mathbf{t}}^{\beta} z(1)=\sigma \mathscr{D}_{\mathbf{t}}^{\beta} z(e)=0,
\end{array}\right.
$$

where $2<\alpha, \beta \leq 3$; $\sigma$ is a differential operator denoted by $t(d / d t)$, that is, $\sigma z(t)=t(d / d t) z(t) ; \mathscr{D}_{\mathbf{t}}^{\alpha}, \mathscr{D}_{\mathbf{t}}^{\beta}$ are the Hadamard fractional derivative of order $\alpha, \beta$; and $f \in(1, e) \times(0$,+ $\infty) \times(0,+\infty),[0,+\infty)$ is a continuous function with singularity at $t=0,1$ and $u=0, v=0$.

Singularity refers to a point or a domain where the given mathematical object is not defined or not "well-behaved." Near a singular point or zone, a minor change of the variable will lead to major changes of the property of the target object. Many physical phenomena in natural sciences and engineering often exhibit some singular behaviour. For example, Fisk
[1] found that in certain materials the quantum fluctuations at absolute zero may push a system into a different phase or state, as result, the process loses its continuity, and then, the singular behaviour happens near the quantum critical points. In fluid mechanics, when a fluid is subjected to a severe impact to form a fracture, singular points or singular domains also follow the fracture. Normally, at singular points and domains, the extreme behaviour such as blow-up phenomena [2,3], impulsive influence [4-9], and chaotic system [10-13], often leads to some difficulties for people in understanding and predicting the corresponding natural problems. Hence, the study of singularity for complex systems governed by differential equations [14-27] is important and interesting in deepening the understanding of the internal laws of dynamic system.

On the other hand, since the fractional differential operator is nonlocal, some often use it to describe viscoelastic behaviour and memory phenomena in various natural science fields such as the silicone gel with the property of weak frequency dependency $[28,29]$ and advection dispersion in anomalous diffusion [30-34]. In most cases, some are interested in the qualitative properties of solutions for the corresponding fractional equations; for the detail, see [35-65]. In 
particular, in order to obtain the qualitative properties of solutions, many nonlinear analysis methods, such as fixed point theorems [66-71], iterative techniques [72-80],variational methods [81-98], and upper and lower solution methods [29, 44], have been developed and employed to study the qualitative properties and numerical results of solutions for various types of differential equations. For example, by using the fixed point index theory, Wang [69] established the existence and multiplicity of positive solutions for the following nonlocal singular fractional differential equation:

$$
\left\{\begin{array}{l}
D_{0_{+}}^{\alpha} u(t)+f(t, u(t)=0,0<t<1, n-1<\alpha \leq n, \\
u(0)=u^{\prime}(0)==u^{(n-2)}(0)=0, D_{0_{+}}^{\beta} u(1)=\int_{0}^{\eta} a(t) D_{0_{+}}^{\gamma} u(t) d V(t),
\end{array}\right.
$$

where $D_{0_{+}}^{\alpha}$ denotes the standard Riemann-Liouville fractional derivative, $\quad n \geq 3,0<\beta<1,0 \leq \gamma<\alpha-1, \eta \in(0,1], f(t, x)$ may be singular at $t=0,1$, and $x=0, a(t) \in L^{1}[0,1] \cap C(0$, 1) is nonnegative. In recent years, to enrich the theory of fractional calculus, the fractional derivative and integral were extended to many different forms such as Hadamard, Erdelyi-Kober, Hilfer derivatives, and integrals. In particular, it is more difficult to obtain the qualitative properties of solutions for Hadamard-type fractional differential equations since Hadamard derivatives possess a singular logarithmic kernel [99-106].

In this paper, we focus on the existence of positive solutions for the Hadamard-type fractional differential equation (1) with singularity in space variables. Our work has some new contributions. Firstly, the equation contains a Hadamard-type fractional derivative which has a singular logarithmic kernel. Secondly, the nonlinearity can have strong singularity in time and space variables. Thirdly, a new limit condition of integral type is introduced to overcome the difficulty of singularity. The rest of this paper is organized as follows. In Section 2, we firstly introduce the concept of Hadamard fractional integral and differential operators and then give the logarithmic kernel and Green function of the boundary value problem and their properties. Our main results are summarized in Section 3.

\section{Preliminaries and Lemmas}

Before the main results, we firstly recall the definition of the Hadamard-type fractional integrals and derivatives; for detail, see [107].

Let $\alpha \in \mathbb{C}, \mathbf{R} e(\alpha)>0, n=[\mathbf{R} e(\alpha)]$ and $(a, b)$ be a finite or infinite interval of $\mathbb{R}^{+}$. The $\alpha$-order left Hadamard fractional integral is defined by

$$
\left(I_{a}^{\alpha} x\right)(t)=\frac{1}{\Gamma(\alpha)} \int_{a}^{t}\left(\ln \frac{t}{s}\right)^{\alpha-1} \frac{x(s)}{s} d s, t \in(a, b),
$$

and the $\alpha$ left Hadamard fractional derivative is defined by

$$
\left(\mathscr{D}_{\mathbf{t}}^{\alpha}\right) x(t)=\frac{1}{\Gamma(n-\alpha)}\left(t \frac{d}{d t}\right)^{n} \int_{a}^{t}\left(\ln \frac{t}{s}\right)^{n-\alpha-1} \frac{x(s)}{s} d s, t \in(a, b) .
$$

In what follows, we consider the following linear auxiliary problem:

$$
\left\{\begin{array}{l}
-\mathscr{D}_{\mathbf{t}}^{\beta} z(t)=x(t), 1<t<e \\
z(1)=\sigma z(1)=\sigma z(e)=0
\end{array}\right.
$$

It follows from [99] that problem (5) has a unique solution

$$
z(t)=\int_{1}^{e} H(t, s) x(s) \frac{d s}{s}
$$

where

$H(t, s)=\frac{1}{\Gamma(\beta)} \begin{cases}(\ln t)^{\beta-1}(1-\ln s)^{\beta-2}-(\ln t-\ln s)^{\beta-1}, & 1 \leq s \leq t \leq e, \\ (\ln t)^{\beta-1}(1-\ln s)^{\beta-2}, & 1 \leq t \leq s \leq e,\end{cases}$

is Green's function of equation (5). Now, let $x(t)=-\mathscr{D}_{\mathbf{t}}^{\beta} z(t)$, then the Hadamard-type fractional differential equation (1) reduces to the following convenient form:

$$
\left\{\begin{array}{l}
-\mathscr{D}_{\mathrm{t}}^{\alpha} x(t)=f\left(t, \int_{1}^{e} H(t, s) x(s) \frac{d s}{s}, x(t)\right), t \in(1, e), \\
x(1)=\sigma z(1)=\sigma x(e)=0 .
\end{array}\right.
$$

It follows from (6) that equation (8) is equivalent to the following integral equation:

$$
x(t)=\int_{1}^{e} G(t, s)\left[f\left(s, \int_{1}^{e} H(s, \tau) x(\tau) \frac{d \tau}{\tau}, x(s)\right)\right] \frac{d s}{s},
$$

where

$$
G(t, s)=\frac{1}{\Gamma(\alpha)} \begin{cases}(\ln t)^{\alpha-1}(1-\ln s)^{\alpha-2}-(\ln t-\ln s)^{\alpha-1}, & 1 \leq s \leq t \leq e \\ (\ln t)^{\alpha-1}(1-\ln s)^{\alpha-2}, & 1 \leq t \leq s \leq e\end{cases}
$$

As a result, in order to find the positive solutions of equation (1), it is sufficient to search the fixed point of the following operator:

$$
T x(t)=\int_{1}^{e} G(t, s)\left[f\left(s, \int_{1}^{e} H(s, \tau) x(\tau) \frac{d \tau}{\tau}, x(s)\right)\right] \frac{d s}{s} .
$$

Lemma 1 (see [99]). Let $\psi_{i}(t)=\ln t(1-\ln t)^{i-2}, i=\alpha, \beta, t \in$ $1, e]$. Then, Green's functions $H, G$ has the following properties: 


$$
H, G \in C\left([1, e] \times[1, e], \mathbb{R}^{+}\right)
$$

(i) For all $t, s \in[1, e]$, the following inequalities hold:

$$
\begin{gathered}
\frac{1}{\Gamma(\beta)}(\ln t)^{\beta-1} \psi_{\beta}(s) \leq H(t, s) \leq \frac{1}{\Gamma(\beta)} \psi_{\beta}(s), \\
\frac{1}{\Gamma(\alpha)}(\ln t)^{\alpha-1} \psi_{\alpha}(s) \leq G(t, s) \leq \frac{1}{\Gamma(\alpha)} \psi_{\alpha}(s)
\end{gathered}
$$
define

Let $Q$ be a cone of Banach space E, for any $0<r<R<\infty$,

$$
\begin{aligned}
\bar{Q}_{r} & =\{x \in Q:\|x\|<r\}, \partial Q_{r}=\{x \in Q:\|x\|=r\}, \bar{Q}_{R} \backslash Q_{r} \\
& =\{x \in Q: r \leq\|x\| \leq R\} .
\end{aligned}
$$

Now, we state the following lemmas which will be used in the rest of the paper.

Lemma 2 (see [108]). Assume $T: \bar{Q}_{r} \rightarrow Q$ is a completely continuous operator.

(i) If there exists $x_{0} \in Q \backslash\{\theta\}$ such that

$$
x-T x \neq \mu x_{0}, x \in \partial Q_{r}, \mu \geq 0,
$$

then, the fixed point index $i\left(T, Q_{r}, Q\right)=0$

(ii) If

$$
T x \neq \mu x, x \in \partial Q_{r}, \mu \geq 1,
$$

then, the fixed point index $i\left(T, Q_{r}, Q\right)=1$

Lemma 3 (Krein-Rutmann, see [108]). Let $L: E \rightarrow E$ be a continuous linear operator, $P$ be a total cone, and $L(P) \subset P$. If there exist $\psi \in E \backslash(-P)$ and a positive constant $c$ such that $c L(\psi) \geq \psi$, then the spectral radius $\rho(L) \neq 0$ and has a positive eigenfunction corresponding to its first eigenvalue $\lambda=\rho(L)^{-1}$.

Lemma 4 (Gelfand's formula, see [108]). For a bounded linear operator $L$ and the operator norm $\|\cdot\|$, the spectral radius of $L^{n}$ satisfies

$$
\rho(L)=\lim _{n \rightarrow+\infty}\left\|L^{n}\right\|^{1 / n}
$$

In this paper, we use the following assumption:
(B1) $f \in C((0,1) \times(0,+\infty) \times(0,+\infty),[0,+\infty))$ and for any $0<r<R<+\infty$,

$$
\lim _{n \rightarrow+\infty} \sup _{u, v \in \bar{Q}_{R} Q_{r}} \int_{\Omega_{n}} \psi_{\alpha}(s) f(s, u(s), v(s)) d s=0,
$$

where $\Omega_{n}=[1,1+(1 / n)] \cup[e-(1 / n), e]$.

Now, let $E=C[1, e]$, then $E$ is a Banach space equipped with the norm $\|x\|=\max _{t \in[1, e]}|x(t)|$. Let $P=\{x \in E: x(t)$ $\geq 0, t \in[1, e]\}$ and

$$
Q=\left\{x \in P: x(t) \geq(\ln t)^{\alpha-1}\|x\|, t \in[1, e]\right\},
$$

then, $Q$ is a cone in the Banach space $E$ and $\bar{Q}_{R} Q_{r} \subset Q \subset P$. Let us define a nonlinear operator $T: \bar{Q}_{R} \backslash Q_{r} \rightarrow P$ and a linear operator $L: E \rightarrow E$ :

$$
\begin{aligned}
& (T x)(t)=\int_{1}^{e} G(t, s)\left[f\left(s, \int_{1}^{e} H(s, \tau) x(\tau) \frac{d \tau}{\tau}, x(s)\right)\right] \frac{d s}{s}, t \in[1, e], \\
& (L x)(t)=\int_{1}^{e} G(t, s) x(s) \frac{d s}{s}, t \in[1, e] .
\end{aligned}
$$

Thus, in order to solve equation (1), we only need to find the fixed point of operator equation $x=T x$. To do this, we firstly establish some lemmas.

Lemma 5. $L: Q \rightarrow Q$ is a completely continuous operator with the spectral radius $\rho(L) \neq 0$. Moreover, $L$ has a positive eigenfunction $\omega^{*}$ corresponding to the first eigenvalue $\mu_{1}=$ $(\rho(L))^{-1}$.

Proof. Firstly, it follows from Lemma 2 that, for any $x \in Q$, one has

$$
\begin{gathered}
\|L x\|=\max _{t \in[1, e]} \int_{1}^{e} G(t, s) x(s) \frac{d s}{s} \leq \frac{1}{\Gamma(\alpha)} \int_{1}^{e} \psi_{\alpha}(s) x(s) \frac{d s}{s}, \\
L x(t) \geq \frac{1}{\Gamma(\alpha)}(\ln t)^{\alpha-1} \int_{1}^{e} \psi_{\alpha}(s) x(s) \frac{d s}{s}
\end{gathered}
$$

which imply that $L: Q \rightarrow Q$. Since $H(t, s), t \in[1, e] \times[1, e]$ is uniform continuity, then the operator $L: Q \rightarrow Q$ is completely continuous.

On the other hand, by (10), we know that there exists a $\tau_{0} \in(1, e)$ such that $G\left(\tau_{0}, \tau_{0}\right)>0$. Thus, from the continuity of $G$, there exists a closed interval $[c, d] \subset(1, e)$ such that $\tau_{0} \in(c, d)$ and $G(t, s)>0$ for all $t, s \in[c, d]$. Now, we take $x \in Q$ such that $x\left(\tau_{0}\right)>0$ and $x(t)=0$ for all $t \in[c, d]$. Then, for all $t \in[c, d]$, one has

$$
(L x)(t)=\int_{1}^{e} G(t, s) x(s) \frac{d s}{s} \geq \int_{c}^{d} G(t, s) x(s) \frac{d s}{s}>0 .
$$

Thus, there exists $\mu>0$ such that $\mu(L x)(t) \geq x(t)$ for $t \in[0,1]$. From Krein-Rutmann's theorem, we know that 
the spectral radius $\rho(L) \neq 0$ and $T$ have a positive eigenfunction $\omega^{*}$ that satisfies $\mu_{1} L \omega^{*}=\omega^{*}$, where $\mu_{1}=(\rho(L))^{-1}$ is its first eigenvalue. The proof is completed.

Lemma 6. Suppose that (B1) holds, then the operator $T: \bar{Q}_{R}$ $\backslash Q_{r} \rightarrow Q$ is completely continuous.

Proof. Firstly, for any $x \in \bar{Q}_{R} \backslash Q_{r}, t \in[1, e]$, it follows from Lemma 2 that

$$
\begin{aligned}
(T x)(t) & =\int_{1}^{e} G(t, s)\left[f\left(s, \int_{1}^{e} H(s, \tau) x(\tau) \frac{d \tau}{\tau}, x(s)\right)\right] \frac{d s}{s} \\
& \leq \frac{1}{\Gamma(\alpha)} \int_{1}^{e} \psi_{\alpha}(s)\left[f\left(s, \int_{1}^{e} H(s, \tau) x(\tau) \frac{d \tau}{\tau}, x(s)\right)\right] \frac{d s}{s}
\end{aligned}
$$

that is

$$
\|T x\| \leq \frac{1}{\Gamma(\alpha)} \int_{1}^{e} \psi_{\alpha}(s)\left[f\left(s, \int_{1}^{e} H(s, \tau) x(\tau) \frac{d \tau}{\tau}, x(s)\right)\right] \frac{d s}{s} .
$$

Similarly, one also has

$$
\begin{aligned}
(T x)(t) & =\int_{1}^{e} G(t, s)\left[f\left(s, \int_{1}^{e} H(s, \tau) x(\tau) \frac{d \tau}{\tau}, x(s)\right)\right] \frac{d s}{s} \\
& \geq \frac{1}{\Gamma(\alpha)}(\ln t)^{\alpha-1} \int_{1}^{e} \psi_{\alpha}(s)\left[f\left(s, \int_{1}^{e} H(s, \tau) x(\tau) \frac{d \tau}{\tau}, x(s)\right)\right] \frac{d s}{s} \\
& \geq(\ln t)^{\alpha-1}\|T x\|,
\end{aligned}
$$

which implies $T(Q) \subset Q$ and then $T\left(\bar{Q}_{R} \backslash Q_{r}\right) \subset Q$.

On the other hand, from (B1), we know that there exists a natural number $l$ such that

$$
\sup _{x \in \bar{Q}_{R} \backslash Q_{r}} \int_{\Omega_{l}} \psi_{\alpha}(s)\left[f\left(s, \int_{1}^{e} H(s, \tau) x(\tau) \frac{d \tau}{\tau}, x(s)\right)\right] \frac{d s}{s}<\frac{1}{2} .
$$

Thus, for any $x \in \bar{Q}_{R} Q_{r}$ and $1+(1 / l) \leq t \leq e-1 / l$, we have

$$
\begin{gathered}
\left(\ln \left(1+\frac{1}{l}\right)\right)^{\alpha-1} r \leq x(t) \leq\|x\|=R, t \in[1, e], \\
\frac{(\ln (1+(1 / l)))^{\alpha-1}(\ln (1+(1 / l)))^{\beta-1} r}{(\beta-1) \Gamma(\beta+1)} \leq \frac{(\ln t)^{\alpha-1}(\ln t)^{\beta-1}}{\Gamma(\beta)} \\
\cdot \int_{1}^{e} \psi_{\beta}(\tau) x(\tau) \frac{d \tau}{\tau}\|x\| \leq \int_{1}^{e} H(s, \tau) x(\tau) \frac{d \tau}{\tau} \\
\leq \frac{1}{\Gamma(\beta)} \int_{1}^{e} \psi_{\beta}(\tau) x(\tau) \frac{d \tau}{\tau} \leq \frac{R}{(\beta-1) \Gamma(\beta+1)}, t \in[1, e] .
\end{gathered}
$$

Take

$$
M_{1}=\max _{(t, x, y) \in \Omega_{l}^{*}}\{f(t, x, y)\}
$$

where

$$
\begin{aligned}
\Omega_{l}^{*}= & {\left[1+\frac{1}{l}, e-\frac{1}{l}\right] \times\left[\frac{(\ln (1+(1 / l)))^{\alpha-1}(\ln (1+(1 / l)))^{\beta-1} R}{(\beta-1) \Gamma(\beta+1)},\right.} \\
& \left.\frac{r}{(\beta-1) \Gamma(\beta+1)}\right] \times\left[\left(\ln \left(1+\frac{1}{l}\right)\right)^{\alpha-1} r, R\right] .
\end{aligned}
$$

So, it follows from (26), (27) and (28) that

$$
\begin{aligned}
\sup _{x \in \bar{Q}_{R} \backslash Q_{r}} \int_{1}^{e} \psi_{\alpha}(s)\left[f\left(s, \int_{1}^{e} H(s, \tau) x(\tau) \frac{d \tau}{\tau}, x(s)\right)\right] \frac{d s}{s} \\
\leq \sup _{x \in \bar{Q}_{R} \backslash Q_{r}} \int_{\Omega_{l}} \psi_{\alpha}(s)\left[f\left(s, \int_{1}^{e} H(s, \tau) x(\tau) \frac{d \tau}{\tau}, x(s)\right)\right] \frac{d s}{s} \\
\quad+\sup _{x \in \bar{Q}_{R} \backslash Q_{r}} \int_{1+1 / l}^{e-1 / l} \psi_{\alpha}(s)\left[f\left(s, \int_{1}^{e} H(s, \tau) x(\tau) \frac{d \tau}{\tau}, x(s)\right)\right] \frac{d s}{s} \\
\leq \frac{1}{2}+M_{1} \int_{1}^{e} \psi_{\alpha}(s) \frac{d s}{s}<+\infty,
\end{aligned}
$$

which implies $T$ is uniformly bounded for any bounded set.

Secondly, we shall prove that $T: \bar{Q}_{R} \backslash Q_{r} \rightarrow Q$ is continuous. To do this, let $x_{n}, x_{0} \in \bar{Q}_{R} Q_{r}$ and $\left\|x_{n}-x_{0}\right\| \rightarrow 0$ $(n \rightarrow \infty)$. For any $\varepsilon>0$, it follows from (B1) that there exists a natural number $m>0$ such that

$$
\sup _{x \in \bar{Q}_{R} \backslash Q_{r}} \int_{\Omega_{m}} \psi_{\alpha}(s)\left[f\left(s, \int_{1}^{e} H(s, \tau) x(\tau) \frac{d \tau}{\tau}, x(s)\right)\right] \frac{d s}{s}<\frac{\varepsilon}{4} .
$$

On the other hand, it follows form the fact that $f(t, u, v)$ is uniformly continuous on $\Omega_{m}^{*}=[1+(1 / m), e-1 / m] \times[((\ln (1$ $\left.+(1 / m)))^{\alpha-1}(\ln (1+(1 / m)))^{\beta-1} R /(\beta-1) \Gamma(\beta+1)\right),(r /(\beta-$ 1) $\Gamma(\beta+1))] \times\left[(\ln (1+(1 / m)))^{\alpha-1} r, R\right],(21)$ that

$$
\begin{aligned}
& \lim _{n \rightarrow+\infty} \mid f\left(s, \int_{1}^{e} H(s, \tau) x_{n}(\tau) \frac{d \tau}{\tau}, x_{n}(s)\right) \\
& -f\left(s, \int_{1}^{e} H(s, \tau) x_{0}(\tau) \frac{d \tau}{\tau}, x_{0}(s)\right) \mid=0,
\end{aligned}
$$

holds uniformly on $s \in[1+(1 / m), e-(1 / m)]$. Thus, the 
Lebesgue control convergence theorem ensures

$$
\begin{aligned}
& \int_{1+(1 / m)}^{e-(1 / m)} \psi_{\alpha}(s) \mid f\left(s, \int_{1}^{e} H(s, \tau) x_{n}(\tau) \frac{d \tau}{\tau}, x_{n}(s)\right) \\
& \quad-f\left(s, \int_{1}^{e} H(s, \tau) x_{0}(\tau) \frac{d \tau}{\tau}, x_{0}(s)\right) \mid \frac{d s}{s} \rightarrow 0, \text { as } n \rightarrow \infty .
\end{aligned}
$$

In other words, for the above $\varepsilon>0$, there exists a positive integer $N$ such that $n>N$, we have

$$
\begin{gathered}
\int_{1+1 / m}^{e-1 / m} \psi_{\alpha}(s) \mid f\left(s, \int_{1}^{e} H(s, \tau) x_{n}(\tau) \frac{d \tau}{\tau}, x_{n}(s)\right) \\
-f\left(s, \int_{1}^{e} H(s, \tau) x_{0}(\tau) \frac{d \tau}{\tau}, x_{0}(s)\right) \mid \frac{d s}{s}<\frac{\varepsilon}{2} .
\end{gathered}
$$

Thus, by (32) and (35), for any $n>N$, we have

$$
\begin{aligned}
& \left\|T x_{n}-T x_{0}\right\| \leq 2 \sup _{x \in \bar{Q}_{R} \backslash Q_{r}} \int_{\Omega_{m}} \psi_{\alpha}(s)\left[f\left(s, \int_{1}^{e} H(s, \tau) x_{0}(\tau) \frac{d \tau}{\tau}, x_{0}(s)\right)\right] \frac{d s}{s} \\
& +\int_{1+(1 / \mathrm{m})}^{e-(1 / m)} \psi_{\alpha}(s) \mid f\left(s, \int_{1}^{e} H(s, \tau) x_{n}(\tau) \frac{d \tau}{\tau}, x_{n}(s)\right) \\
& \quad-f\left(s, \int_{1}^{e} H(s, \tau) x_{0}(\tau) \frac{d \tau}{\tau}, x_{0}(s)\right) \mid \frac{d s}{s}<2 \times \frac{\varepsilon}{4}+\frac{\varepsilon}{2}=\varepsilon .
\end{aligned}
$$

Therefore, $T: \bar{Q}_{R} Q_{r} \rightarrow Q$ is continuous.

In the end, we shall prove that $T$ is equicontinuous. Firstly, it follows from (B1) that for any $\varepsilon>0$, there exists a positive integer $k$ such that

$$
\sup _{x \in \bar{Q}_{R} \backslash Q_{r}} \int_{\Omega_{k}} \psi_{\alpha}(s)\left[f\left(s, \int_{1}^{e} H(s, \tau) x(\tau) \frac{d \tau}{\tau}, x(s)\right)\right] \frac{d s}{s}<\frac{\varepsilon}{4} .
$$

Take

$$
M_{2}=\max _{(t, x, y) \in \Omega_{k}^{*}}\{f(t, x, y)\}
$$

where

$$
\begin{aligned}
\Omega_{k}^{*}= & {\left[1+\frac{1}{k}, e-\frac{1}{k}\right] \times\left[\frac{(\ln (1+1 / k))^{\alpha-1}(\ln (1+(1 / k)))^{\beta-1} r}{(\beta-1) \Gamma(\beta+1)},\right.} \\
& \left.\frac{R}{(\beta-1) \Gamma(\beta+1)}\right] \times\left[\left(\ln \left(1+\frac{1}{k}\right)\right)^{\alpha-1} r, R\right] .
\end{aligned}
$$

Notice that $G(t, s)$ is uniformly continuous on $[1, e] \times$ $[1, e]$, so for the above $\varepsilon>0$ and fixed $s \in[1+(1 / k), e-(1 / k)]$, there exists $\delta>0$, when $\left|t_{1}-t_{2}\right|<\delta, t_{1}, t_{2} \in[1, e]$, we have

$$
\left|G\left(t_{1}, s\right)-G\left(t_{2}, s\right)\right| \leq\left[2 M_{2}(e-1)\right]^{-1} \varepsilon .
$$

It follows from the above argument that, for $\left|t_{1}-t_{2}\right|<\delta$, $t_{1}, t_{2} \in[1, e]$, one has

$$
\begin{aligned}
& \left|T x(t)-T x\left(t^{\prime}\right)\right| \leq 2 \sup _{x \in \bar{Q}_{R} \backslash Q_{r}} \int_{\Omega_{k}} \psi_{\alpha}(s)\left[f\left(s, \int_{1}^{e} H(s, \tau) x(\tau) \frac{d \tau}{\tau}, x(s)\right)\right] \frac{d s}{s} \\
& +\sup _{x \in \bar{Q}_{R} \backslash Q_{r}} \int_{1+(1 / k)}^{e-(1 / k)}\left|G(t, s)-G\left(t^{\prime}, s\right)\right|\left[f\left(s, \int_{1}^{e} H(s, \tau) x(\tau) \frac{d \tau}{\tau}, x(s)\right)\right] \frac{d s}{s} \\
& \quad<2 \times \frac{\varepsilon}{4}+\frac{\varepsilon}{2}=\varepsilon,
\end{aligned}
$$

which implies that $T$ is an equicontinuous operator. According to the Arzela-Ascoli Theorem, $T: \bar{Q}_{R} \backslash Q_{r} \rightarrow Q$ is completely continuous. The proof is completed.

\section{Main Results}

We state the main results of this paper as follows.

Theorem 7. Let $\mu_{1}$ be the first eigenvalue of L, assume (B1) holds and

$$
\limsup _{\substack{u+v \rightarrow+\infty \\ v \rightarrow+\infty}} \frac{f(t, u, v)}{v}<\mu_{1}<\liminf _{\substack{u \rightarrow 0^{+} \\ v \rightarrow 0^{+}}} \frac{f(t, u, v)}{u+v}
$$

uniformly holds on $t \in[1, e]$. Then, equation (1) has at least one positive solution.

Proof. Firstly, by Lemma 6, we know that $T: \bar{Q}_{R} \backslash Q_{r} \rightarrow Q$ is completely continuous. Thus, it follows from the extension theorem of a completely continuous operator (see Theorem 8.3 on page 56 of [108]) that, for any $R>0$, there exists a completely continuous operator $T^{*}: \bar{Q}_{R} \rightarrow Q$. Thus, without loss of the generality, we shall still write it as $T$.

Next, it follows from (42) that there exists $r>0$ such that

$$
f(t, u, v) \geq \mu_{1}(u+v),|v| \leq \frac{r}{(\beta-1) \Gamma(\beta+1)},|x| \leq r, t \in[1, e] .
$$

Since, for any $x \in \partial Q_{r}$, we have

$$
\left|\int_{1}^{e} H(s, \tau) x(\tau) \frac{d \tau}{\tau}\right| \leq \frac{r}{(\beta-1) \Gamma(\beta+1)},|x(s)| \leq r,
$$

thus, for $x \in \partial Q_{r}$, from (43) and (44), one gets

$$
\begin{aligned}
(T x)(t) & =\int_{1}^{e} G(t, s)\left[f\left(s, \int_{1}^{e} H(s, \tau) x(\tau) \frac{d \tau}{\tau}, x(s)\right)\right] \frac{d s}{s} \\
& \geq \mu_{1} \int_{1}^{e} G(t, s)\left|\int_{1}^{e} H(s, \tau) x(\tau) \frac{d \tau}{\tau}+x(s)\right| \frac{d s}{s} \\
& \geq \mu_{1}(L x)(t), t \in[1, e] .
\end{aligned}
$$

Let $\omega^{*}$ be the positive eigenfunction corresponding to $\mu_{1}$, 
i.e., $\omega^{*}=\mu_{1} L \omega^{*}$. In what follows, we shall use the contradiction method to show

$$
x-T x \neq \mu \omega^{*}, x \in \partial Q_{r}, \mu \geq 0 .
$$

Firstly, we can suppose that $T$ has no fixed points on $x$ $\in \partial Q_{r}$ (otherwise, the proof is finished). In this case, let us suppose there exist $x_{0} \in \partial Q_{r}$ and $\mu_{0} \geq 0$ such that $x_{0}-T x_{0}$ $=\mu_{0} \omega^{*}$. This implies that $\mu_{0}>0$ and then

$$
x_{0}=T x_{0}+\mu_{0} \omega^{*} \geq \mu_{0} \omega^{*}
$$

Let $\bar{\mu}=\sup \left\{\mu \mid x_{0} \geq \mu \omega^{*}\right\}$, then $\bar{\mu} \geq \mu_{0}, x_{0} \geq \bar{\mu} \omega^{*}, \mu_{1} L x_{0}$ $\geq \mu_{1} \bar{\mu} L \omega^{*}=\bar{\mu} \omega^{*}$. Thus, it follows from (45) that

$x_{0}=T x_{0}+\mu_{0} \omega^{*} \geq \mu_{1} L x_{0}+\mu_{0} \omega^{*} \geq \bar{\mu} \omega^{*}+\mu_{0} \omega^{*}=\left(\bar{\mu}+\mu_{0}\right) \omega^{*}$,

which contradicts with the definition of $\bar{\mu}$. So (46) holds and from Lemma 2, we have

$$
i\left(T, Q_{r}, Q\right)=0 \text {. }
$$

On the other hand, it follows from (42) that there exists $R_{1}>r$ and $0<\kappa<1$ such that

$$
f(t, u, v) \leq \kappa \mu_{1}|v|, \text { for }|u+v| \geq R_{1},|v| \geq R_{1} .
$$

Let $\tilde{L} x=\kappa \mu_{1} L x$. Obviously, $\tilde{L}: E \rightarrow E$ is also a bounded linear operator and $\tilde{L}(Q) \subset Q$. Since $\mu_{1}$ is the first eigenvalue of operator $L$ and $0<\kappa<1$, we have

$$
r^{-1}(\tilde{L})=\left(\kappa \mu_{1} r(L)\right)^{-1}=\kappa^{-1}>1
$$

From Gelfand's formula, we have

$$
\kappa=\lim _{n \rightarrow+\infty}\left\|\tilde{L}^{n}\right\|^{1 / n}
$$

Now, choose $\varepsilon_{0}=1 / 2(1-\kappa)$, it follows from (52) that there exists an enough large integer $N>0$ such that when $n$ $\geq N$, one has

$$
\left\|\tilde{L}^{n}\right\| \leq\left[\kappa+\varepsilon_{0}\right]^{n}
$$

Let $\tilde{L}^{0}=I$ be the identity operator and define

$$
\|x\|^{*}=\sum_{i=1}^{N}\left[\kappa+\varepsilon_{0}\right]^{N-i}\left\|\tilde{L}^{i-1} x\right\|, x \in E,
$$

then $\|\cdot\|^{*}$ is still the norm of $E$. Let

$$
M=\sup _{x \in \partial Q_{R_{1}}} \int_{1}^{e} \psi_{\alpha}(s)\left[f\left(s, \int_{1}^{e} H(s, \tau) x(\tau) \frac{d \tau}{\tau}, x(s)\right)\right] \frac{d s}{s},
$$

then from (31), we have $M<+\infty$. Take

$$
R_{2}>\max \left\{R_{1}, \frac{2}{\varepsilon_{0}} M^{*}\right\}
$$

where $M^{*}=\|M\|^{*}$. Notice that $\|x\|^{*}>\left[\kappa+\varepsilon_{0}\right]^{N-1}\|x\|$, so we can choose a large enough $R>R_{2}$ such that $\|x\| \geq R$ implies $\|x\|^{*}>R_{2}$.

Next, we shall show

$$
T x \neq \mu x, x \in \partial Q_{R}, \mu \geq 1 \text {. }
$$

Otherwise, there exist $x_{1} \in \partial Q_{R}$ and $\mu^{*} \geq 1$ such that $T$ $x_{1}=\mu^{*} x_{1}$. Let $\tilde{x}(t)=\min \left\{x_{1}(t), R_{1}\right\}$ and

$$
D\left(x_{1}\right)=\left\{t \in[1, e]: x_{1}(t)>R_{1}\right\} \text {. }
$$

Since $x_{1} \in C[1, e], x_{1}(t) \leq\left\|x_{1}\right\|=R$, there exists $1<t_{0} \leq e$ such that $x_{1}\left(t_{0}\right)=R$. So for any $t \in[1, e]$, we get $\tilde{x}(t)=\min$ $\left\{x_{1}(t), R_{1}\right\} \leq \min \left\{R, R_{1}\right\}=R_{1}$ and $\tilde{x}\left(t_{0}\right)=\min \left\{x_{1}\left(t_{0}\right), R_{1}\right\}$ $=\min \left\{R, R_{1}\right\}=R_{1}$, which implies $\|\tilde{x}(t)\|=R_{1}$, i.e., $\tilde{x} \in \partial$ $Q_{R_{1}}$. Thus by Lemma 2, we have

$$
\begin{aligned}
\mu^{*} x_{1}= & \left(T x_{1}\right)(t)=\int_{1}^{e} G(t, s)\left[f\left(s, \int_{1}^{e} H(s, \tau) x_{1}(\tau) \frac{d \tau}{\tau}, x_{1}(s)\right)\right] \frac{d s}{s} \\
\leq & \int_{D\left(x_{1}\right)} G(t, s)\left[f\left(s, \int_{1}^{e} H(s, \tau) x_{1}(\tau) \frac{d \tau}{\tau}, x_{1}(s)\right)\right] \frac{d s}{s} \\
& +\int_{[1, e] \backslash D\left(x_{1}\right)} \psi_{\alpha}(s)\left[f\left(s, \int_{1}^{e} H(s, \tau) \tilde{x}(\tau) \frac{d \tau}{\tau}, \tilde{x}(s)\right)\right] \frac{d s}{s} \\
\leq & \kappa \mu_{1} \int_{1}^{e} G(t, s) x_{1}(s) \frac{d s}{s}+\int_{1}^{e} \psi_{\alpha}(s)\left[f\left(s, \int_{1}^{e} H(s, \tau) \tilde{x}(\tau) \frac{d \tau}{\tau}, \tilde{x}(s)\right)\right] \frac{d s}{s} \\
\leq & \left(\tilde{L} x_{1}\right)(t)+M, t \in[1, e] .
\end{aligned}
$$

So it follows from $\tilde{L}(Q) \subset Q$ and (59), we have

$0 \leq\left(\tilde{L}^{j}\left(T x_{1}\right)\right)(t) \leq\left(\tilde{L}^{j}\left(\tilde{L} x_{1}+M\right)\right)(t), j=0,1,2, \cdots, N-1$

Since $Q$ is a normal cone with normality constant 1 and (60), one has

$$
\left\|\tilde{L}^{j}\left(T x_{1}\right)\right\| \leq\left\|\tilde{L}^{j}\left(\tilde{L} x_{1}+M\right)\right\|, j=0,1,2, \cdots, N-1,
$$

which leads to

$$
\begin{aligned}
\left\|T x_{1}\right\|^{*} & =\sum_{i=1}^{N}\left[\kappa+\varepsilon_{0}\right]^{N-i}\left\|\tilde{L}^{i-1}\left(T x_{1}\right)\right\| \\
& \leq \sum_{i=1}^{N}\left[\kappa+\varepsilon_{0}\right]^{N-i}\left\|\tilde{L}^{i-1}\left(\tilde{L} x_{1}+M\right)\right\|=\left\|\tilde{L} x_{1}+M\right\|^{*} .
\end{aligned}
$$

According to the selection of $R_{2}$, we have $M^{*}<\left(\varepsilon_{0} / 2\right) R_{2}$. Thus, it follows from the fact $\|x\| \geq R$ implies $\|x\|^{*}>R_{2}$ and 
(53), (54), and (62) that

$$
\begin{aligned}
\mu^{*}\left\|x_{1}\right\|^{*}= & \left\|T x_{1}\right\|^{*} \leq\left\|\tilde{L} x_{1}\right\|^{*}+M^{*}=\sum_{i=1}^{N}\left[\kappa+\varepsilon_{0}\right]^{N-i}\left\|\tilde{L}^{i} x_{1}\right\|+M^{*} \\
= & {\left[\kappa+\varepsilon_{0}\right] \sum_{i=1}^{N-1}\left[\kappa+\varepsilon_{0}\right]^{N-i-1}\left\|\tilde{L}^{i} x_{1}\right\|+\left\|\tilde{L}^{N} x_{1}\right\|+M^{*} } \\
\leq & {\left[\kappa+\varepsilon_{0}\right] \sum_{i=1}^{N-1}\left[\kappa+\varepsilon_{0}\right]^{N-i-1}\left\|\tilde{L}^{i} x_{1}\right\|+\left[\kappa+\varepsilon_{0}\right]^{N}\left\|x_{1}\right\|+M^{*} } \\
= & {\left[\kappa+\varepsilon_{0}\right] \sum_{i=1}^{N}\left[\kappa+\varepsilon_{0}\right]^{N-i}\left\|\tilde{L}^{i-1} x_{1}\right\|+M^{*} \leq\left[\kappa+\varepsilon_{0}\right]\left\|x_{1}\right\|^{*} } \\
& +\frac{\varepsilon_{0}}{2} R_{2} \leq\left[\kappa+\varepsilon_{0}\right]\left\|x_{1}\right\|^{*}+\frac{\varepsilon_{0}}{2}\left\|x_{1}\right\|^{*}=\left[\frac{1}{4} \kappa+\frac{3}{4}\right]\left\|x_{1}\right\|^{*} .
\end{aligned}
$$

Notice that $\mu^{*} \geq 1$, we have $(1 / 4) \kappa+3 / 4 \geq 1$, and then $\kappa$ $\geq 1$, which is a contradiction with $0<\kappa<1$. So (57) is indeed valid and it follows from Lemma 2 that

$$
i\left(T, Q_{R}, Q\right)=1 .
$$

Thus, (49) and (64) lead to

$$
i\left(T, Q_{R} \backslash \bar{Q}_{r}, Q\right)=i\left(T, Q_{R}, Q\right)-i\left(T, Q_{r}, Q\right)=1,
$$

which implies $T$ has at least one fixed point on $Q_{R} \backslash \bar{Q}_{r}$. Consequently, equation (1) has at least one positive solution.

Theorem 8. Let $\mu_{1}$ be the first eigenvalue of $L$, suppose (B1) holds, and

$$
\limsup _{\substack{u \rightarrow 0^{+} \\ v \rightarrow 0^{+}}} \frac{f(t, u, v)}{v}<\mu_{1}, \quad \liminf _{u+v \rightarrow+\infty} \frac{f(t, u, v)}{u+v}>\tilde{\mu}_{1}
$$

uniformly on $t \in[1, e]$, where $\tilde{\mu}_{1}$ is any eigenvalues of $L$. Then, equation (1) has at least one positive solution.

To prove Theorem 8 , we need some preliminaries and lemma. For any enough small $0<\varepsilon<1$, define

$$
\left(L_{\varepsilon} x\right)(t)=\int_{1+\varepsilon}^{e-\varepsilon} G(t, s) x(s) \frac{d s}{s}, t \in[1, e] .
$$

By Lemma 5, we know $L_{\varepsilon}: Q \rightarrow Q$ is still a completely continuous linear operator with the spectral radius $\rho\left(L_{\varepsilon}\right) \neq$ 0 , and $L_{\varepsilon}$ has a positive eigenfunction $\omega_{\varepsilon}$ corresponding to its first eigenvalue $\mu_{\varepsilon}=\left(\rho\left(L_{\varepsilon}\right)\right)^{-1}$.

Lemma 9. There exists an eigenvalue $\tilde{\mu}_{1}$ of $L$ such that

$$
\lim _{\varepsilon \rightarrow 0^{+}} \mu_{\varepsilon}=\tilde{\mu}_{1} \text {. }
$$

Proof. Let $\varepsilon_{m} \rightarrow 0(m \rightarrow \infty)$ satisfying $\varepsilon_{1} \geq \varepsilon_{2} \geq \cdots \geq \varepsilon_{m} \geq \cdots$. Thus, for $\omega \in Q$ and $k>m$, one has

$$
\left(L_{\varepsilon_{m}} \omega\right)(t) \leq\left(L_{\varepsilon_{k}} \omega\right)(t) \leq(L \omega)(t), \quad t \in[1, e],
$$

and then

$$
\left(L_{\varepsilon_{m}}^{n} \omega\right)(t) \leq\left(L_{\varepsilon_{k}}^{n} \omega\right)(t) \leq\left(L^{n} \omega\right)(t), \quad t \in[1, e], n=2,3, \cdots,
$$

where $L_{\varepsilon_{m}}^{n}=L\left(L_{\varepsilon_{m}}^{n-1}\right), n=2,3, \cdots$. It follows from the fact that $Q$ is a normal cone with normality constant 1 that

$$
\left\|L_{\varepsilon_{m}}^{n}\right\| \leq\left\|L_{\varepsilon_{k}}^{n}\right\| \leq\left\|L^{n}\right\|, n=1,2, \cdots .
$$

By Gelfand's formula, we have $\mu_{\varepsilon_{m}} \geq \mu_{\varepsilon_{k}} \geq \mu_{1}$, where $\mu_{1}$ is the first eigenvalue of $L$, which implies $\left\{\mu_{\varepsilon_{m}}\right\}$ is monotonous with lower boundedness $\mu_{1}$. Let

$$
\lim _{m \rightarrow+\infty} \mu_{\varepsilon_{m}}=\tilde{\mu}_{1}
$$

we assert $\tilde{\mu}_{1}$ is an eigenvalue of $L$.

In fact, let $\omega_{\varepsilon_{m}}$ be positive eigenfunctions of $L_{\varepsilon_{m}}$ corresponding to $\mu_{\varepsilon_{m}}$ with $\left\|\omega_{\varepsilon_{m}}\right\|=1, m=1,2, \cdots$. So we have

$\omega_{\varepsilon_{m}}(t)=\mu_{\varepsilon_{m}} L_{\varepsilon_{m}} \omega_{\varepsilon_{m}}(t)=\mu_{\varepsilon_{m}} \int_{1+\varepsilon_{m}}^{e-\varepsilon_{m}} G(t, s) \omega_{\varepsilon_{m}}(s) \frac{d s}{s}, \quad t \in[1, e]$

Since

$$
\begin{aligned}
\left\|L_{\varepsilon_{m}} \omega_{\varepsilon_{m}}\right\| & =\max _{1 \leq t \leq e} \int_{1+\varepsilon_{m}}^{e-\varepsilon_{m}} G(t, s) \omega_{\varepsilon_{m}}(s) \frac{d s}{s} \leq \frac{1}{\Gamma(\alpha)} \int_{1}^{e} \psi_{\alpha}(s) \frac{d s}{s} \\
& <+\infty,(m=1,2, \cdots),
\end{aligned}
$$

$\left\{L_{\varepsilon_{m}} \omega_{\varepsilon_{m}}\right\} \subset Q$ is uniformly bounded.

Moreover, for any $m$ and $t_{1}, t_{2} \in[1, e]$, one has

$\left|L_{\varepsilon_{m}} \omega_{\varepsilon_{m}}\left(t_{1}\right)-L_{\varepsilon_{m}} \omega_{\varepsilon_{m}}\left(t_{2}\right)\right| \leq \int_{\varepsilon_{m}}^{1-\varepsilon_{m}}\left|G\left(t_{1}, s\right)-G\left(t_{2}, s\right)\right| \omega_{\varepsilon_{m}}(s) \frac{d s}{s}$.

Notice that $G(t, s)$ is uniformly continuous on $[1, e] \times$ $[1, e]$; then, (75) implies $\left\{L_{\varepsilon_{m}} \omega_{\varepsilon_{m}}\right\} \subset Q$ is equicontinuous. Thus, from the Arzela-Ascoli theorem and $\lim _{m \rightarrow+\infty} \mu_{\varepsilon_{m}}=$ $\tilde{\mu}_{1}$, we know $\omega_{\varepsilon_{m}} \rightarrow \omega_{0}$ as $m \rightarrow+\infty$, which yields $\left\|\omega_{0}\right\|=1$. Take the limit for two sides of (73), we obtain

$$
\omega_{0}(t)=\tilde{\mu}_{1} \int_{1}^{e} G(t, s) \omega_{0}(s) \frac{d s}{s}, \quad t \in[1, e],
$$

that is, $\omega_{0}=\tilde{\mu}_{1} L \omega_{0}$, and $\tilde{\mu}_{1}$ is an eigenvalue of $L$. 
Proof of Theorem 8. Firstly, it follows from (66) that for any $t \in[1, e]$, there exists $r>0$ such that

$$
f(t, u, v) \leq \mu_{1} v,|u| \leq \frac{r}{(\beta-1) \Gamma(\beta+1)},|v| \leq r .
$$

Thus, for any $x \in \partial Q_{r}$, since

$$
\left|\int_{1}^{e} H(s, \tau) x(\tau) \frac{d \tau}{\tau}\right| \leq \frac{r}{(\beta-1) \Gamma(\beta+1)},|x(s)| \leq\|x\|=r,
$$

we have

$$
\begin{aligned}
(T x)(t) & =\int_{1}^{e} G(t, s)\left[f\left(s, \int_{1}^{e} H(s, \tau) x(\tau) \frac{d \tau}{\tau}, x(s)\right)\right] \frac{d s}{s} \\
& \leq \mu_{1} \int_{1}^{e} G(t, s) x(s) \frac{d s}{s}=\mu_{1}(L x)(t), t \in[1, \mathrm{e}] .
\end{aligned}
$$

In the following, we prove that

$$
T x \neq \mu x \text {, for any } x \in \partial Q_{r}, \mu \geq 1 \text {. }
$$

Firstly, we may suppose that $T$ has no fixed point on $\partial Q_{r}$ (otherwise, the proof is completed). If (80) is not true, i.e., there exist $x_{0} \in \partial Q_{r}$ and $\mu_{0} \geq 1$ satisfying $T x_{0}=\mu_{0} x_{0}$. Thus $\mu_{0}>1$, it follows from (79) that

$$
\mu_{0} x_{0}=T x_{0} \leq \mu_{1} L x_{0} .
$$

From induction, we have

$$
\mu_{0}^{n} x_{0} \leq \mu_{1}^{n} L^{n} x_{0}, n=1,2, \cdots
$$

which implies that

$$
\left\|L^{n}\right\| \geq \frac{\left\|L^{n} x_{0}\right\|}{\left\|x_{0}\right\|} \geq \frac{\mu_{0}^{n}\left\|x_{0}\right\|}{\mu_{1}^{n}\left\|x_{0}\right\|}=\frac{\mu_{0}^{n}}{\mu_{1}^{n}} .
$$

Thus, by Gelfand's formula, we have

$$
r(L)=\lim _{n \rightarrow \infty} \sqrt[n]{\left\|L^{n}\right\|} \geq \frac{\mu_{0}}{\mu_{1}}>\frac{1}{\mu_{1}},
$$

which contradicts with $r(L)=\mu_{1}^{-1}$. Therefore, (80) is valid and from Lemma 2, we get

$$
i\left(T, Q_{r}, Q\right)=1
$$

On the other hand, for any fixed small enough $0<\varepsilon<1$, take

$$
\rho_{\varepsilon}=\frac{\Gamma(\alpha-1)(\ln (1+\varepsilon))^{\beta-1}}{(\beta-1) \Gamma(\alpha+\beta-2)}+(\ln (1+\varepsilon))^{\alpha-1} .
$$

By (66) and $\lim _{\varepsilon \rightarrow 0^{+}} \mu_{\varepsilon}=\tilde{\mu}_{1}$, we can find a sufficiently small $\varepsilon>0$ and $R>r$ such that

$$
f(t, u, v) \geq \mu_{\varepsilon}(u+v), u+v \geq \rho_{\varepsilon} R, t \in[1, e],
$$

where $\mu_{\varepsilon}$ is the first eigenvalue of $L_{\varepsilon}$.

Let $\omega_{\varepsilon}$ be the positive eigenfunction of $L_{\varepsilon}$ corresponding to $\mu_{\varepsilon}$, which implies $\omega_{\varepsilon}=\mu_{\varepsilon} L_{\varepsilon} \omega_{\varepsilon}$. For any $x \in \partial Q_{R}, s \in[1+\varepsilon$, $e-\varepsilon]$, we have

$$
\begin{aligned}
& \int_{1}^{e} H(s, \tau) x(\tau) \frac{d \tau}{\tau}+x(s) \geq \frac{1}{\Gamma(\beta)}(\ln s)^{\beta-1} \int_{1}^{e} \psi_{\beta}(\tau)(\ln \tau)^{\alpha-1} \frac{d \tau}{\tau}\|x\| \\
& +(\ln s)^{\alpha-1}\|x\| \geq\left(\frac{\Gamma(\alpha-1)(\ln (1+\varepsilon))^{\beta-1}}{(\beta-1) \Gamma(\alpha+\beta-2)}+(\ln (1+\varepsilon))^{\alpha-1}\right) R=\rho_{\varepsilon} R .
\end{aligned}
$$

Thus, it follows from (87) and (88) that

$$
\begin{aligned}
(T x)(t) & =\int_{1}^{e} G(t, s)\left[f\left(s, \int_{1}^{e} H(s, \tau) x(\tau) \frac{d \tau}{\tau}, x(s)\right)\right] \frac{d s}{s} \\
& \geq \int_{1+\varepsilon}^{e-\varepsilon} G(t, s)\left[f\left(s, \int_{1}^{e} H(s, \tau) x(\tau) \frac{d \tau}{\tau}, x(s)\right)\right] \frac{d s}{s} \\
& \geq \mu_{\varepsilon} \int_{1+\varepsilon}^{e-\varepsilon} G(t, s)\left(\int_{1}^{e} H(s, \tau) x(\tau) \frac{d \tau}{\tau}+x(s)\right) \frac{d s}{s} \\
& \geq \mu_{\varepsilon} \int_{1+\varepsilon}^{e-\varepsilon} G(t, s) x(s) \frac{d s}{s}=\mu_{\varepsilon}\left(L_{\varepsilon} x\right)(t), t \in[1, e] .
\end{aligned}
$$

Similar to the proof of Theorem 7, we obtain

$$
x-T x \neq \mu \omega_{\varepsilon}, x \in \partial Q_{R}, \mu \geq 0 .
$$

According to Lemma 2, we have

$$
i\left(T, Q_{R}, Q\right)=0
$$

Combining (80) and (91), one has

$$
i\left(T, Q_{R} \backslash \bar{Q}_{r}, Q\right)=i\left(T, Q_{R}, Q\right)-i\left(T, Q_{r}, Q\right)=-1 .
$$

Hence, $T$ has at least one fixed point on $Q_{R} \backslash \bar{Q}_{r}$, and then equation (1) has at least a positive solution. The proof is completed.

\section{Conclusion}

Singular behaviour is a class of important natural phenomena in many physical science, mathematics, engineering, and bioscience. So, the study for singularity is an interesting and challenging problem. In this paper, we consider the existence of positive solutions for a Hadamard-type fractional differential equation with singular nonlinearity by introducing a new limit-type growth condition. The main advantage of the assumption is that it provides an effective method for handling the singularity at space variables. This assumption is valid and reasonable and easier to get the solution of the target equation. 


\section{Data Availability}

Not applicable.

\section{Conflicts of Interest}

The authors declare that there is no conflict of interest regarding the publication of this paper.

\section{Authors' Contributions}

The study was carried out by the collaboration of all authors. All authors read and approved the final manuscript.

\section{Acknowledgments}

The authors are supported financially by the National Natural Science Foundation of China (11871302, 11571296)

\section{References}

[1] Z. Fisk, "Condensed-matter physics: singular behaviour," Nature, vol. 424, no. 6948, pp. 504-505, 2003.

[2] X. Zhang, Y. Wu, and Y. Cui, "Existence and nonexistence of blow-up solutions for a Schrödinger equation involving a nonlinear operator," Applied Mathematics Letters, vol. 82, pp. 85-91, 2018.

[3] X. Zhang, L. Liu, Y. Wu, and Y. Cui, "A sufficient and necessary condition of existence of blow-up radial solutions for a k-Hessian equation with a nonlinear operator," Nonlinear Analysis: Modelling and Control, vol. 25, no. 1, pp. 126-143, 2020.

[4] L. Ren, J. Wang, and M. Feckan, "Periodic mild solutions of impulsive fractional evolution equations," AIMS Mathematics, vol. 5, no. 1, pp. 497-506, 2019.

[5] B. Zhang, Y. Xia, L. Zhu, H. Liu, and L. Gu, "Global stability of fractional order coupled systems with impulses via a graphic approach,” Mathematics, vol. 7, no. 8, p. 744, 2019.

[6] J. R. Wang, A. G. Ibrahim, and D. O'Regan, "Global attracting solutions to Hilfer fractional differential inclusions of Sobolev type with noninstantaneous impulses and nonlocal conditions," Nonlinear Analysis: Modelling and Control, vol. 24, no. 5, pp. 775-803, 2019.

[7] J. R. Wang and M. Feckan, "Periodic solutions and stability of linear evolution equations with noninstantaneous impulses," Miskolc Mathematical Notes, vol. 20, no. 2, pp. 1299-1313, 2019.

[8] Y. Chen and J. Wang, "Continuous dependence of solutions of integer and fractional order non-instantaneous impulsive equations with random impulsive and junction points," Mathematics, vol. 7, no. 4, p. 331, 2019.

[9] J. Wang, A. G. Ibrahim, and D. O'Regan, "Nonemptyness and compactness of the solution set for fractional evolution inclusions with non-instantaneous impulses," Electronic Journal of Differential Equations, vol. 2019, no. 37, pp. 1-17, 2019.

[10] S. Xu, H. Lv, H. Liu, and A. Liu, "Robust control of disturbed fractional-order economical chaotic systems with uncertain parameters," Complexity, vol. 2019, Article ID 7567695, 13 pages, 2019.

[11] M. Fečkan, T. Sathiyaraj, and J. R. Wang, "Synchronization of butterfly fractional order chaotic system," Mathematics, vol. 8 , no. 3, p. 446, 2020.
[12] F. Wang and Z. Zheng, "Quasi-projective synchronization of fractional order chaotic systems under input saturation," Physica A: Statistical Mechanics and its Applications, vol. 534, p. 122132, 2019.

[13] S. Ha, H. Liu, S. Li, and A. Liu, "Backstepping-based adaptive fuzzy synchronization control for a class of fractional-order chaotic systems with input saturation," International Journal of Fuzzy Systems, vol. 21, no. 5, pp. 1571-1584, 2019.

[14] F. Yan, M. Zuo, and X. Hao, "Positive solution for a fractional singular boundary value problem with $p$-Laplacian operator," Boundary Value Problems, vol. 2018, no. 1, 2018.

[15] J. Liu and Z. Zhao, "Existence of positive solutions to a singular boundary-value problem using variational methods," Electronic Journal of Differential Equations, vol. 2014, no. 135 , pp. 1-9, 2014

[16] X. Zhang, L. Liu, Y. Wu, and B. Wiwatanapataphee, "The spectral analysis for a singular fractional differential equation with a signed measure," Applied Mathematics and Computation, vol. 257, pp. 252-263, 2015.

[17] L. Liu, F. Sun, X. Zhang, and Y. Wu, "Bifurcation analysis for a singular differential system with two parameters via to topological degree theory," Nonlinear Analysis: Modelling and Control, vol. 2017, no. 1, pp. 31-50, 2017.

[18] Y. Wang, "Existence and multiplicity of positive solutions for a class of singular fractional nonlocal boundary value problems," Boundary Value Problems, vol. 2019, no. 1, 2019.

[19] F. Wang, L. Liu, and Y. Wu, "A numerical algorithm for a class of fractional BVPs with $p$-Laplacian operator and singularity-the convergence and dependence analysis," Applied Mathematics and Computation, vol. 382, article 125339, 2020.

[20] S. Song, B. Zhang, X. Song, Y. Zhang, Z. Zhang, and W. Li, "Fractional-order adaptive neuro-fuzzy sliding mode $H_{\infty}$ control for fuzzy singularly perturbed systems," Journal of the Franklin Institute, vol. 356, no. 10, pp. 5027-5048, 2019.

[21] X. Zhang, C. Mao, L. Liu, and Y. Wu, "Exact iterative solution for an abstract fractional dynamic system model for bioprocess," Qualitative Theory of Dynamical Systems, vol. 16, no. 1, pp. 205-222, 2017.

[22] F. Wang, L. Liu, and Y. Wu, "Iterative unique positive solutions for a new class of nonlinear singular higher order fractional differential equations with mixed-type boundary value conditions," Journal of Inequalities and Applications, vol. 2019, no. 1, 2019.

[23] T. Ren, H. Xiao, Z. Zhou et al., "The iterative scheme and the convergence analysis of unique solution for a singular fractional differential equation from the eco-economic complex System's co-evolution process," Complexity, vol. 2019, Article ID 9278056, 15 pages, 2019.

[24] J. Wu, X. Zhang, L. Liu, Y. Wu, and Y. Cui, “The convergence analysis and error estimation for unique solution of a $p$ Laplacian fractional differential equation with singular decreasing nonlinearity," Boundary Value Problems, vol. 2018, no. 1, Article ID 82, 2018.

[25] X. Zhang, L. Yu, J. Jiang, Y. Wu, and Y. Cui, "Positive solutions for a weakly singular Hadamard-type fractional differential equation with changing-sign nonlinearity," Journal of Function Spaces, vol. 2020, Article ID 5623589, 10 pages, 2020.

[26] X. Zhang, L. Liu, and Y. Wu, "The uniqueness of positive solution for a singular fractional differential system involving 
derivatives," Communications in Nonlinear Science and Numerical Simulation, vol. 18, no. 6, pp. 1400-1409, 2013.

[27] X. Zhang, L. Liu, and Y. Wu, "The eigenvalue problem for a singular higher order fractional differential equation involving fractional derivatives," Applied Mathematics and Computation, vol. 218, no. 17, pp. 8526-8536, 2012.

[28] H. Schiessel, R. Metzler, A. Blumen, and T. F. Nonnenmacher, "Generalized viscoelastic models: their fractional equations with solutions," Journal of Physics A: Mathematical and General, vol. 28, no. 23, pp. 6567-6584, 1995.

[29] J. He, X. Zhang, L. Liu, Y. Wu, and Y. Cui, “A singular fractional Kelvin-Voigt model involving a nonlinear operator and their convergence properties," Boundary Value Problems, vol. 2019, no. 1, 2019.

[30] X. Zhang, L. Liu, and Y. Wu, "Variational structure and multiple solutions for a fractional advection-dispersion equation," Computers \& Mathematcs with Applications, vol. 68, no. 12, pp. 1794-1805, 2014.

[31] B. Zhu, L. Liu, and Y. Wu, "Existence and uniqueness of global mild solutions for a class of nonlinear fractional reaction-diffusion equations with delay," Computers \& Mathematics with Applications, vol. 78, no. 6, pp. 1811-1818, 2019.

[32] J. Zhao, Y. Zhang, and Y. Xu, "Implicit Runge-Kutta and spectral Galerkin methods for Riesz space fractional/distributed-order diffusion equation," Computational and Applied Mathematics, vol. 39, no. 2, p. 47, 2020.

[33] X. Zhang, L. Liu, Y. Wu, and B. Wiwatanapataphee, "Nontrivial solutions for a fractional advection dispersion equation in anomalous diffusion," Applied Mathematics Letters, vol. 66, pp. 1-8, 2017.

[34] D. Ma, L. Liu, and Y. Wu, "Existence of nontrivial solutions for a system of fractional advection-dispersion equations," Revista de la Real Academia de Ciencias Exactas, Físicas y Naturales. Serie A. Matemáticas, vol. 113, no. 2, pp. 1041-1057, 2019.

[35] K. Liu, M. Fečkan, D. O’Regan, and J. R. Wang, "Hyers-Ulam stability and existence of solutions for differential equations with Caputo-Fabrizio fractional derivative," Mathematics, vol. 7, no. 4, p. 333, 2019.

[36] J. Mao, Z. Zhao, and C. Wang, "The exact iterative solution of fractional differential equation with nonlocal boundary value conditions," Journal of Function Spaces, vol. 2018, Article ID 8346398, 6 pages, 2018.

[37] M. Li and J. Wang, "Exploring delayed Mittag-Leffler type matrix functions to study finite time stability of fractional delay differential equations," Applied Mathematics and Computation, vol. 324, pp. 254-265, 2018.

[38] M. Feckan, M. Pospisil, and J. Wang, "Note on weakly fractional differential equations," Advances in Difference Equations, vol. 2019, no. 1, 2019.

[39] Q. Feng and F. Meng, "Traveling wave solutions for fractional partial differential equations arising in mathematical physics by an improved fractional Jacobi elliptic equation method," Mathematicsl Methods in the Applied Sciences, vol. 40, no. 10, pp. 3676-3686, 2017.

[40] M. Li and J. Wang, "Representation of solution of a RiemannLiouville fractional differential equation with pure delay," Applied Mathematics Letters, vol. 85, pp. 118-124, 2018.

[41] F. Wang and Y. Yang, "Quasi-synchronization for fractionalorder delayed dynamical networks with heterogeneous nodes," Applied Mathematics and Computation, vol. 339, pp. 1-14, 2018.
[42] Y. Wang and L. Liu, "Positive solutions for a class of fractional infinite-point boundary value problems," Boundary Value Problems, vol. 2018, no. 1, 2018.

[43] Y. Wang, "Positive solutions for a class of two-term fractional differential equations with multipoint boundary value conditions," Advances in Difference Equations, vol. 2019, no. $1,2019$.

[44] J. He, X. Zhang, L. Liu, Y. Wu, and Y. Cui, "Existence and asymptotic analysis of positive solutions for a singular fractional differential equation with nonlocal boundary conditions," Boundary Value Problems, vol. 2018, no. 1, 2018.

[45] X. Hao, H. Wang, L. Liu, and Y. Cui, "Positive solutions for a system of nonlinear fractional nonlocal boundary value problems with parameters and p-Laplacian operator," Boundary Value Problems, vol. 2017, no. 1, 2017.

[46] T. Ren, S. Li, X. Zhang, and L. Liu, "Maximum and minimum solutions for a nonlocal p-Laplacian fractional differential system from eco-economical processes," Boundary Value Problems, vol. 2017, no. 1, 2017.

[47] X. Zhang, L. Liu, and Y. Wu, "Multiple positive solutions of a singular fractional differential equation with negatively perturbed term," Mathematical and Computer Modelling, vol. 55, no. 3-4, pp. 1263-1274, 2012.

[48] Y. Wang and L. Liu, "Positive solutions for a class of fractional 3-point boundary value problems at resonance," Advances in Difference Equations, vol. 2017, no. 1, 2017.

[49] Y. Wang and L. Liu, "Necessary and sufficient condition for the existence of positive solution to singular fractional differential equations," Advances in Difference Equations, vol. 2015, no. 1, 2015.

[50] X. Zhang, L. Liu, B. Wiwatanapataphee, and Y. Wu, "The eigenvalue for a class of singular $p$-Laplacian fractional differential equations involving the Riemann-Stieltjes integral boundary condition," Applied Mathematics and Computation, vol. 235, pp. 412-422, 2014.

[51] Y. Wang, "Necessary conditions for the existence of positive solutions to fractional boundary value problems at resonance," Applied Mathematics Letters, vol. 97, pp. 34-40, 2019.

[52] X. Zhang, L. Liu, Y. Wu, and Y. Cui, "New result on the critical exponent for solution of an ordinary fractional differential problem," Journal of Function Spaces, vol. 2017, Article ID 3976469, 4 pages, 2017.

[53] M. Ahmad, J. Jiang, A. Zada, S. O. Shah, and J. Xu, "Analysis of coupled system of implicit fractional differential equations involving Katugampola-Caputo fractional derivative," Complexcity, vol. 2020, article 9285686, 11 pages, 2020.

[54] H. Liu and R. Xu, "The oscillatory of linear conformable fractional differential equations of Kamenev type," Discrete Dynamics in Nature and Society, vol. 2020, Article ID 3857592, 8 pages, 2020.

[55] Z. Zheng, H. Liu, J. Cai, and Y. Zhang, "Criteria of limit-point case for conformable fractional Sturm-Liouville operators," Mathematical Methods in the Applied Sciences, vol. 43, no. 5, pp. 2548-2557, 2020.

[56] X. Wang, J. R. Wang, and M. Fečkan, "Controllability of conformable differential systems," Nonlinear Analysis: Modelling and Control, vol. 25, no. 4, pp. 658-674, 2020.

[57] F. Sun, L. Liu, X. Zhang, and Y. Wu, "Spectral analysis for a singular differential system with integral boundary conditions," Mediterranean Journal of Mathematics, vol. 13, pp. 4763-4782, 2016. 
[58] J. Wu, X. Zhang, L. Liu, Y. Wu, and Y. Cui, "Convergence analysis of iterative scheme and error estimation of positive solution for a fractional differential equation," Mathematical Modelling and Analysis, vol. 23, pp. 611-626, 2018.

[59] X. Zhang, L. Liu, and Y. Wu, "The uniqueness of positive solution for a fractional order model of turbulent flow in a porous medium," Applied Mathematics Letters, vol. 37, pp. 26-133, 2014.

[60] Y. Wang, L. Liu, X. Zhang, and Y. Wu, "Positive solutions of an abstract fractional semipositone differential system model for bioprocesses of HIV infection," Applied Mathematics and Computation, vol. 258, pp. 312-1324, 2015.

[61] J. Zhao, Y. Zhang, and Y. Xu, "Implicit Runge-Kutta and spectral Galerkin methods for the two-dimensional nonlinear Riesz space fractional diffusion equation," Applied Mathematics and Computation, vol. 386, article 125505, 2020.

[62] Q. Zhao and S. Zhao, "Constructing minimum aberration split-plot designs via complementary sets when the whole plot factors are important," Journal of Statistical Planning and Inference, vol. 209, pp. 123-143, 2020.

[63] J. Zhao, Y. Zhang, and Y. Xu, "Implicit Runge-Kutta and spectral Galerkin methods for the two-dimensional nonlinear Riesz space distributed-order diffusion equation," Applied Numerical Mathematics, vol. 157, pp. 223-235, 2020.

[64] L. Liu, D. Min, and Y. Wu, "Existence and multiplicity of positive solutions for a new class of singular higher-order fractional differential equations with Riemann-Stieltjes integral boundary value conditions," Advances in Difference Equations, vol. 2020, no. 1, Article ID 442, 2020.

[65] T. Wang and Z. Hao, "Existence and uniqueness of positive solutions for singular nonlinear fractional differential equation via mixed monotone operator method," Journal of Function Spaces, vol. 2020, Article ID 2354927, 9 pages, 2020.

[66] J. Wang, A. Zada, and H. Waheed, "Stability analysis of a coupled system of nonlinear implicit fractional antiperiodic boundary value problem," Mathematical Methods in the Applied Sciences, vol. 42, no. 18, pp. 6706-6732, 2019.

[67] Z. You, M. Feckan, and J. Wang, "Relative controllability of fractional delay differential equations via delayed perturbation of Mittag-Leffler functions," Journal of Computational and Applied Mathematics, vol. 378, p. 112939, 2020.

[68] X. Zhang, J. Jiang, Y. Wu, and Y. Cui, "Existence and asymptotic properties of solutions for a nonlinear Schrödinger elliptic equation from geophysical fluid flows," Applied Mathematics Letters, vol. 90, pp. 229-237, 2019.

[69] X. Zhang and L. Liu, "Existence results for multiple positive solutions of nonlinear higher order perturbed fractional differential equations with derivatives," Applied Mathematics and Computation, vol. 216, pp. 1420-1433, 2012.

[70] J. He, X. Zhang, L. Liu, and Y. Wu, "Existence and nonexistence of radial solutions of the Dirichlet problem for a class of general $k$-Hessian equations," Nonlinear Analysis: Modelling and Control, vol. 23, no. 4, pp. 475-492, 2018.

[71] P. Yang, J. Wang, and M. Feckan, "Periodic nonautonomous differential equations with noninstantaneous impulsive effects," Mathematical Methods in the Applied Sciences, vol. 42, no. 10, pp. 3700-3720, 2019.

[72] J. Mao, Z. Zhao, and C. Wang, "The unique iterative positive solution of fractional boundary value problem with q-difference," Applied Mathematics Letters, vol. 100, p. 106002, 2020.
[73] Y. Cui and Y. Zou, "Monotone iterative method for differential systems with coupled integral boundary value problems," Boundary Value Problems, vol. 2013, no. 1, 2013.

[74] K. Pei, G. Wang, and Y. Sun, "Successive iterations and positive extremal solutions for a Hadamard type fractional integro-differential equations on infinite domain," Applied Mathematics and Computation, vol. 312, pp. 158-168, 2017.

[75] X. Zhang, L. Liu, and Y. Wu, "The entire large solutions for a quasilinear Schrödinger elliptic equation by the dual approach," Applied Mathematics Letters, vol. 55, pp. 1-9, 2016.

[76] S. Liu, J. R. Wang, D. Shen, and D. O’Regan, “Iterative learning control for differential inclusions of parabolic type with noninstantaneous impulses," Applied Mathematics and Computation, vol. 350, pp. 48-59, 2019.

[77] S. Liu, J. R. Wang, D. Shen, and D. O'Regan, "Iterative learning control for noninstantaneous impulsive fractional-order systems with varying trial lengths," International Journal of Robust and Nonlinear Control, vol. 28, no. 18, pp. 62026238, 2018.

[78] H. Che, H. Chen, and M. Li, "A new simultaneous iterative method with a parameter for solving the extended split equality problem and the extended split equality fixed point problem," Numerical Algorithms, vol. 79, no. 4, pp. 1231-1256, 2018.

[79] X. Zhang, J. Xu, J. Jiang, Y. Wu, and Y. Cui, “The convergence analysis and uniqueness of blow-up solutions for a Dirichlet problem of the generalk-Hessian equations," Applied Mathematics Letters, vol. 102, article 106124, 2020.

[80] K. Zhang and Y. Wang, "AnH-tensor based iterative scheme for identifying the positive definiteness of multivariate homogeneous forms," Journal of Computational and Applied Mathematics, vol. 305, pp. 1-10, 2016.

[81] J. Liu and Z. Zhao, "Multiple solutions for impulsive problems with non-autonomous perturbations," Applied Mathematics Letters, vol. 64, pp. 143-149, 2017.

[82] A. Mao, R. Jing, S. Luan, J. Chu, and Y. Kong, "Some nonlocal elliptic problem involing positive parameter," Topological Methods in Nonlinear Analysis, vol. 42, pp. 207-220, 2013.

[83] J. Liu and Z. Zhao, "An application of variational methods to second-order impulsive differential equation with derivative dependence," Electronic Journal of Differential Equations, vol. 2014, p. 62, 2014.

[84] A. Mao and W. Wang, "Nontrivial solutions of nonlocal fourth order elliptic equation of Kirchhoff type in $\mathbb{R}^{3}$," Journal of Mathematical Analysis and Applications, vol. 459, no. 1, pp. 556-563, 2018.

[85] M. Shao and A. Mao, "Multiplicity of solutions to SchrödingerPoisson system with concave-convex nonlinearities," Applied Mathematics Letters, vol. 83, pp. 212-218, 2018.

[86] J. Sun, T. Wu, and Z. Feng, "Non-autonomous SchrödingerPoisson system in $\mathbb{R}^{3}$," Discrete \& Continuous Dynamical Systems - A, vol. 38, no. 4, pp. 1889-1933, 2018.

[87] J. Zhang, Z. Lou, Y. Ji, and W. Shao, "Ground state of Kirchhoff type fractional Schrödinger equations with critical growth," Journal of Mathematical Analysis and Applications, vol. 462, no. 1, pp. 57-83, 2018.

[88] X. He, A. Qian, and W. Zou, "Existence and concentration of positive solutions for quasilinear Schrödinger equations with critical growth," Nonlinearity, vol. 26, no. 12, pp. 3137-3168, 2013. 
[89] X. Zhang, L. Liu, Y. Wu, and Y. Cui, "Existence of infinitely solutions for a modified nonlinear Schrödinger equation via dual approach," Electronic Journal of Differential Equations, vol. 147, pp. 1-15, 2018.

[90] A. Mao, Y. Zhu, and S. Luan, "Existence of solutions of elliptic boundary value problems with mixed type nonlinearities," Boundary Value Problems, vol. 2012, no. 1, 2012.

[91] J. Sun and T. Wu, "Steep potential well may help Kirchhoff type equations to generate multiple solutions," Nonlinear Analysis, vol. 190, p. 111609, 2020.

[92] J. Sun and T. Wu, "Bound state nodal solutions for the nonautonomous Schrödinger-Poisson system in $\mathbb{R}^{3}$," Journal of Differential Equations, vol. 268, no. 11, pp. 7121-7163, 2020.

[93] A. Mao and X. Zhu, "Existence and multiplicity results for Kirchhoff problems," Mediterranean Journal of Mathematics, vol. 14, no. 2, p. 58, 2017.

[94] X. Zhang, J. Jiang, Y. Wu, and Y. Cui, “The existence and nonexistence of entire large solutions for a quasilinear Schrödinger elliptic system by dual approach," Applied Mathematics Letters, vol. 100, article 106018, 2020.

[95] X. Zhang, L. Liu, Y. Wu, and Y. Cui, "The existence and nonexistence of entire large solutions for a quasilinear Schrödinger elliptic system by dual approach," Journal of Mathematical Analysis and Applications, vol. 464, no. 2, pp. 10891106, 2018.

[96] X. Zhang, L. Liu, Y. Wu, and Y. Cui, "Entire blow-up solutions for a quasilinear $p$-Laplacian Schrödinger equation with a non-square diffusion term," Applied Mathematics Letters, vol. 74, pp. 85-93, 2017.

[97] X. Zhang, L. Liu, Y. Wu, and L. Caccetta, "Entire large solutions for a class of Schrodinger systems with a nonlinear random operator," Journal of Mathematical Analysis and Applications, vol. 423, pp. 1650-1659, 2015.

[98] X. Zhang, L. Liu, Y. Wu, and Y. Lu, "The iterative solutions of nonlinear fractional differential equations," Applied Mathematics and Computation, vol. 219, no. 9, pp. 4680-4691, 2013.

[99] Y. Ding, J. Jiang, D. O’Regan, and J. Xu, "Positive solutions for a system of Hadamard-type fractional differential equations with semipositone nonlinearities," Complexity, vol. 2020, Article ID 9742418, 14 pages, 2020.

[100] X. Wu, J. Wang, and J. Zhang, "Hermite-Hadamard-type inequalities for convex functions via the fractional integrals with exponential kernel," Mathematics, vol. 7, no. 9, p. 845, 2019.

[101] P. Yang, J. Wang, and Y. Zhou, "Representation of solution for a linear fractional delay differential equation of Hadamard type," Advances in Difference Equations, vol. 2019, no. 1, 2019.

[102] J. Jiang, D. O’Regan, J. Xu, and Y. Cui, “Positive solutions for a Hadamard fractional $p$-Laplacian three-point boundary value problem," Mathematics, vol. 7, no. 5, p. 439, 2019.

[103] K. Liu, J. Wang, and D. O'Regan, "On the HermiteHadamard type inequality for $\psi$-Riemann-Liouville fractional integrals via convex functions," Journal of Inequalities and Applications, vol. 2019, no. 1, 2019.

[104] W. Liu, L. Liu, and Y. Wu, "Existence of solutions for integral boundary value problems of singular Hadamard-type fractional differential equations on infinite interval," Advances in Difference Equations, vol. 2020, no. 1, 2020.
[105] J. Mao, Z. Zhao, and C. Wang, "The unique positive solution for singular Hadamard fractional boundary value problems," Journal of Function Spaces, vol. 2019, Article ID 5923490, 6 pages, 2019.

[106] J. Jiang, D. O'Regan, J. Xu, and Z. Fu, “Positive solutions for a system of nonlinear Hadamard fractional differential equations involving coupled integral boundary conditions," Journal of Inequalities and Applications, vol. 2019, no. 1, 2019.

[107] A. Kilbas, H. Srivastava, and J. Trujillo, Theory and Applications of Fractional Differential Equations, Elsevier, Boston, MA, USA, 2006.

[108] K. Deimling, Nonlinear Functional Analysis, Springer-Verlag, Berlin, Grermany, 1985. 